\title{
A Geometric View of Complex Trigonometric Functions
}

\author{
Richard Hammack
}

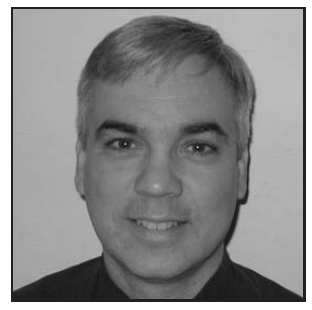

Richard Hammack (rhammack@vcu.edu) received his Ph.D. from the University of North Carolina at Chapel Hill. He developed an early version of this article while an undergraduate art student at Rhode Island School of Design, and it later became part of his master's thesis at Virginia Commonwealth University. Following positions at Wake Forest University and Randolph-Macon College, he is now an associate professor at Virginia Commonwealth University. When not teaching or doing graph theory, he spends time with his wife Micol and young daughters Adriana and Sage.

Students of trigonometry and calculus learn to interpret $\sin \alpha$ and $\cos \alpha$ as the $y$ and $x$ coordinates of a point located by radian measure $\alpha$ on the unit circle. In this geometric setting, the unit circle is a natural protractor that measures angles on the $x-y$ plane, and sine and cosine are functions that resolve angles into vertical and horizontal components.

In more advanced courses, analytic reasoning is used to extend these functions to the complex domain:

$$
\begin{aligned}
& \cos (\alpha+\beta i)=\cos \alpha \cosh \beta-i \sin \alpha \sinh \beta \\
& \sin (\alpha+\beta i)=\sin \alpha \cosh \beta+i \cos \alpha \sinh \beta .
\end{aligned}
$$

(See for instance [3, p. 78].) Visual thinkers may accept this with a degree of reluctance. They may wonder how_or if — these equations could be understood visually. Can they be seen as arising from a complex version of the unit circle? Can an angle with imaginary or complex radian measure be visualized? This article describes how this can be done. We define the sine and cosine of a complex number through purely geometric means, and get (1) and (2) as a consequence.

\section{The complex unit circle}

While trigonometry's familiar unit circle lies in $\mathbb{R}^{2}$, our goal suggests that we investigate the unit circle as a set of points in complex two-dimensional space

$$
\mathbb{C}^{2}=\left\{\left[\begin{array}{c}
z \\
w
\end{array}\right] \mid z, w \in \mathbb{C}\right\}
$$

Let us agree to write $z=x+y i$ and $w=u+v i$, so

$$
\mathbb{C}^{2}=\left\{\left[\begin{array}{l}
x+y i \\
u+v i
\end{array}\right] \mid x, y, u, v \in \mathbb{R}\right\},
$$

which can be identified with Euclidean four-dimensional space. Despite this fourdimensional flavor, $\mathbb{C}^{2}$ is of course a two-dimensional vector space over the field $\mathbb{C}$. 
Its one-dimensional subspaces are all isomorphic to the complex plane. For example, the subspace

$$
\operatorname{span}\left(\left[\begin{array}{l}
1 \\
0
\end{array}\right]\right)=\left\{\left[\begin{array}{c}
x+y i \\
0
\end{array}\right] \mid x, y \in \mathbb{R}\right\}
$$

is clearly a copy of $\mathbb{C}$ inside $\mathbb{C}^{2}$. We call it the $x$-yi plane. Likewise, the $u$-vi plane

$$
\left\{\left[\begin{array}{c}
0 \\
u+v i
\end{array}\right] \mid u, v \in \mathbb{R}\right\}
$$

is another subspace. There are four other "coordinate planes" obtained by setting two of $x, y, u$, and $v$ zero, but none of these is a subspace, as they are not closed under scalar multiplication.

We define the complex unit circle to be the subset

$$
U=\left\{\left[\begin{array}{c}
z \\
w
\end{array}\right] \mid z^{2}+w^{2}=1\right\}
$$

of $\mathbb{C}^{2}$, in a manner exactly analogous to the definition of the standard unit circle in $\mathbb{R}^{2}$. Apparently $U$ is some sort of surface in $\mathbb{C}^{2}$, but it can't be drawn as simply as the usual unit circle, owing to the four-dimensional character of $\mathbb{C}^{2}$. Still, it is not difficult to get a feel for what $U$ looks like.

First, let's examine some of its lower-dimensional cross-sections. By setting $y=$ $v=0$, it is immediately clear that $U$ intersects the $x$ - $u$ plane of $\mathbb{C}^{2}$ at the familiar (real) unit circle $x^{2}+u^{2}=1$. Widening our scope slightly, let's set only $y=0$, and examine how $U$ intersects the $x$ - $u$-vi coordinate space of $\mathbb{C}^{2}$. The definition of $U$ gives $x^{2}+(u+v i)^{2}=1$, or $x^{2}+u^{2}-v^{2}+2 u v i=1$. Since the imaginary part is 0 , either $v$ or $u$ is 0 . In the first case, we get the circle $x^{2}+u^{2}=1$ in the $x$ - $u$ plane. The second case produces the hyperbola $x^{2}-v^{2}=1$ in the $x$-vi plane. These two curves touch at the points $\pm \mathbf{o}$, where $\mathbf{o}=\left[\begin{array}{l}1 \\ 0\end{array}\right]$, as illustrated in Figure 1. (The symbol $\mathbf{o}$ is used because this point will be the origin of complex radian measure.)

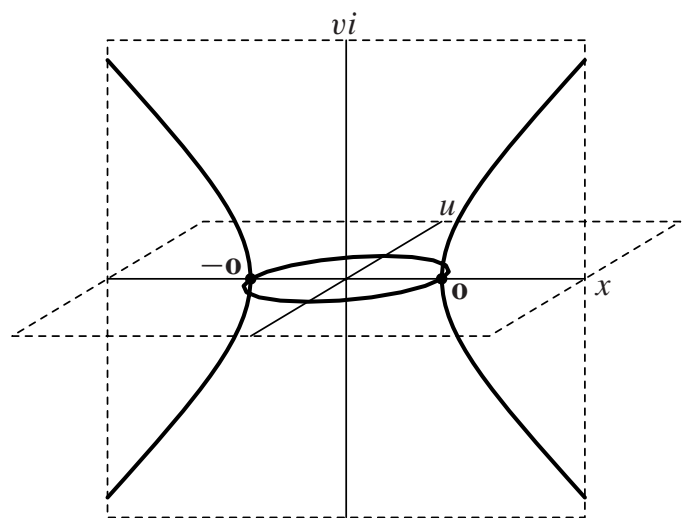

Figure 1. The cross-section of $U$ with the $x$ - $u$-vi coordinate space.

Of course, this is just a cross-section of $U$; the rest of it is "out there" in $\mathbb{C}^{2}$, where $y \neq 0$. We can develop a more complete picture by realizing that the right-hand sides of (1) and (2) parameterize $U$ with complex parameter $\alpha+\beta i$. It is easy to confirm 
that the points

$$
\left[\begin{array}{c}
z \\
w
\end{array}\right]=\left[\begin{array}{l}
\cos \alpha \cosh \beta-i \sin \alpha \sinh \beta \\
\sin \alpha \cosh \beta+i \cos \alpha \sinh \beta
\end{array}\right]
$$

satisfy $z^{2}+w^{2}=1$, so they are on $U$. Conversely, it's easy to check that every point on $U$ has this form. Consequently, the complex unit circle is the image of the function $\mu: \mathbb{C} \rightarrow \mathbb{C}^{2}$ defined as

$$
\mu(\alpha+\beta i)=\left[\begin{array}{l}
\cos \alpha \cosh \beta-i \sin \alpha \sinh \beta \\
\sin \alpha \cosh \beta+i \cos \alpha \sinh \beta
\end{array}\right] .
$$

Holding $\beta=0$, we get $\mu(\alpha)=\left[\begin{array}{l}\cos \alpha \\ \sin \alpha\end{array}\right]$, so $\mu$ maps the real axis of the complex plane onto the (real) unit circle on the $x$ - $u$ plane, as shown in Figure 1. If $\alpha=0$, then $\mu(\beta i)=$ $\left[\begin{array}{c}\cosh \beta \\ i \sinh \beta\end{array}\right]$, so $\mu$ maps the imaginary axis onto the right-hand branch of the hyperbola on the $x$-vi plane, also illustrated in Figure 1. What about the left-hand branch? Since $\mu(\pi+\beta i)=\left[\begin{array}{l}-\cosh \beta \\ -\sinh \beta\end{array}\right]$, that branch is the image of the vertical line $\{\pi+\beta i \mid \beta \in \mathbb{R}\}$ in $\mathbb{C}$. More generally, for any integer $k, \mu$ maps the vertical line $\{k \pi+\beta i \mid \beta \in \mathbb{R}\}$ in $\mathbb{C}$ to the right- or left-hand branch, depending on whether $k$ is even or odd. Thus Figure 1 depicts the image of the real axis of $\mathbb{C}$, together with the images of the vertical lines $\{k \pi+\beta i \mid \beta \in \mathbb{R}\}$.

From the periodic nature of sine and cosine, it follows that $\mu(\alpha+2 k \pi+\beta i)=$ $\mu(\alpha+\beta i)$ for all real $\alpha$ and $\beta$ and any integer $k$. Thus $\mu$ maps the infinite strip $S=\{\alpha+\beta i \mid \alpha, \beta \in \mathbb{R},-\pi \leq \alpha \leq \pi\}$ in the complex plane onto $U$, with $\mu(-\pi+\beta i)=\mu(\pi+\beta i)$ for all $\beta$. Topologically, then, the surface $U$ is like the strip $S$, twisted around in $\mathbb{C}^{2}$, with its left and right edges matching up to form a cylinder.

Figure 2 shows a portion of the strip $S$, together with its image in $U$ projected orthogonally to two-dimensional space. (It is suggested that the reader not be concerned with the exact nature of the projection, but simply use the picture as an aid in under-
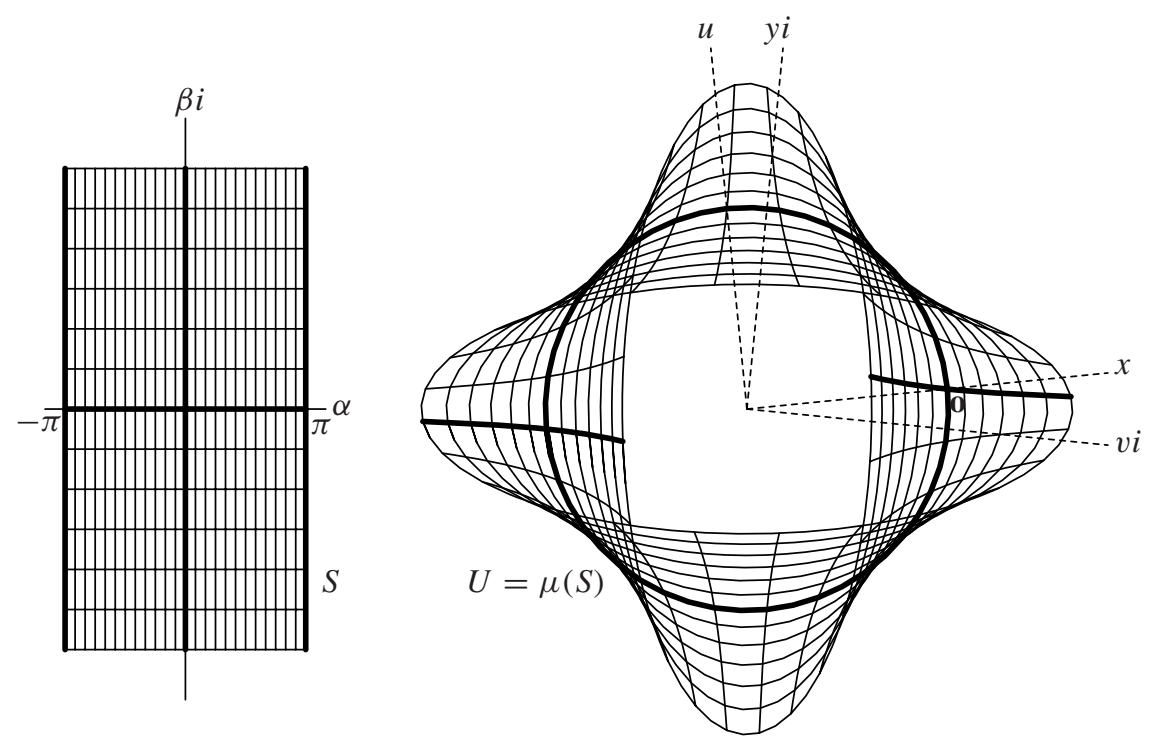

Figure 2. The complex unit circle $U$. 


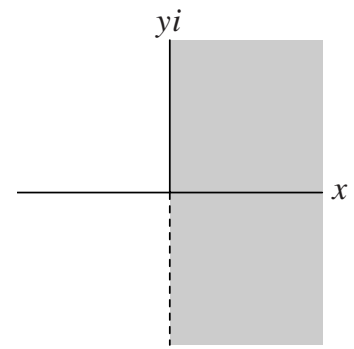

Figure 3. The range $H$ of $\sqrt{z}$.

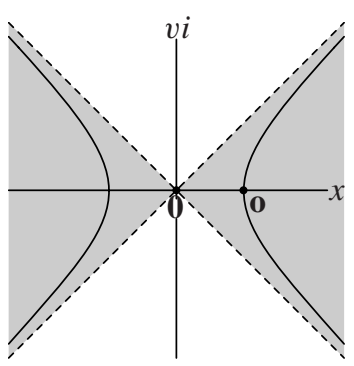

Figure 4. The $x$-vi plane.

standing the character of $U$.) The picture reveals the cylindrical nature of $U$; imagine looking down the axis of a cylinder whose bottom is deformed into a north-south ellipse and whose top is deformed into a east-west ellipse. The map $\mu$ sends horizontal lines on $S$ to ellipses, and vertical lines to hyperbolas. Curves from Figure 1 are shown bold on $U$ in Figure 2.

\section{Complex measurement}

One expects the unit circle to consist of points a unit distance from the origin $\mathbf{0}=\left[\begin{array}{l}0 \\ 0\end{array}\right]$, but that does not, at first, seem to happen with $U$. Points on the hyperbola in Figure 1 appear to be arbitrarily far away from the origin. To overcome this philosophical difficulty, we define distance in $\mathbb{C}^{2}$ in a special way. The complex distance between points $\left[\begin{array}{l}z_{1} \\ w_{1}\end{array}\right]$ and $\left[\begin{array}{c}z_{2} \\ w_{2}\end{array}\right]$ in $\mathbb{C}^{2}$ is defined to be

$$
d\left(\left[\begin{array}{c}
z_{1} \\
w_{1}
\end{array}\right],\left[\begin{array}{c}
z_{2} \\
w_{2}
\end{array}\right]\right)=\sqrt{\left(z_{1}-z_{2}\right)^{2}+\left(w_{1}-w_{2}\right)^{2}}
$$

In this equation (and henceforth, whenever the square root is encountered) we use the branch of the square root function for which $\sqrt{z}$ is in the half-plane $H$ of $\mathbb{C}$ consisting of the non-negative imaginary axis and numbers with a positive real part (see Figure 3).

In general, the complex distance between two points in $\mathbb{C}^{2}$ is a complex number (with non-negative real part). However, since any point $\left[\begin{array}{c}z \\ w\end{array}\right]$ on $U$ satisfies $z^{2}+w^{2}=$ 1 , its complex distance from the origin of $\mathbb{C}^{2}$ is 1 . The complex unit circle $U$ is thus the set of all points in $\mathbb{C}^{2}$ that are at complex distance 1 from the origin.

Several things about complex distance are worth mentioning. First, expression (4) is the exact formula we would use for the Euclidean distance in $\mathbb{R}^{2}$, but we are here applying it to $\mathbb{C}^{2}$. Although it cannot be considered a metric in the technical sense, if we restrict our attention to points in the (real) $x$ - $u$ plane of $\mathbb{C}^{2}$, then complex distance reduces to the familiar Euclidean metric. Thus, the $x-u$ plane is the Euclidean plane, and the circular cross-section of $U$ in the $x$ - $u$ plane is metrically identical to the familiar unit circle.

Next, consider the $x$-vi plane of $\mathbb{C}^{2}$, shown in Figure 4 with the hyperbolic crosssection of $U$. Let $\mathbf{p}=\left[\begin{array}{c}x \\ v i\end{array}\right]$ be a point in this plane. The complex distance from $\mathbf{p}$ to the origin $\mathbf{0}$ is seen to be $\sqrt{x^{2}-v^{2}}$. Depending on $\mathbf{p}$, this can be real, imaginary, or zero (even when $\mathbf{p}$ is nonzero). If $\mathbf{p}$ is on the hyperbola (the intersection of $U$ with the $x$-vi plane), then $x^{2}-v^{2}=1$, so $\mathbf{p}$ is at complex distance 1 from $\mathbf{0}$. If $\mathbf{p}$ is in the 
shaded region, then $x^{2}-v^{2}>0$, so the complex distance from $\mathbf{p}$ to $\mathbf{0}$ is real. For $\mathbf{p}$ in the white region, we have $x^{2}-v^{2}<0$, so its complex distance from $\mathbf{0}$ is purely imaginary. Finally, if $\mathbf{p}$ is on an asymptote, then $x^{2}-v^{2}=0$, so its complex distance from $\mathbf{0}$ is 0 . Similar remarks hold for the $u-y i$ plane (which also contains a hyperbolic cross-section of $U$ ).

Despite its unsettling properties, complex distance is exactly what is needed to make $U$ behave like a complex version of the unit circle. The next two sections show that if we start at the point $\mathbf{o}$ in $U$, and move a complex distance of $\alpha+\beta i$ along $U$ to a point $\mathbf{p}$, then $\cos (\alpha+\beta i)$ and $\sin (\alpha+\beta i)$ are the $z$ and $w$ coordinates of $\mathbf{p}$, respectively.

\section{Real and imaginary radian measure}

Starting at the point $\mathbf{o}$ in Figure 1, one can move either along the circle or along the right-hand branch of the hyperbola. We now investigate these two choices and show that they involve traversing either a real or an imaginary distance. This leads to a model of real and imaginary radian measure in which sine and cosine can be understood geometrically.

First consider moving along the circle on the $x-u$ plane, shown in Figure 5(a). Since the $x-u$ plane is metrically identical to the Euclidean plane, the length of the traversed path can be regarded as the standard (real) radian measure of trigonometry, with the usual convention of counterclockwise and clockwise paths corresponding to positive and negative radian measure. If $\mathbf{p}$ is at distance $\alpha$ from $\mathbf{0}$ along the circle, then $\cos \alpha$ and $\sin \alpha$ are the $z$ and $w$ coordinates of $\mathbf{p}$.

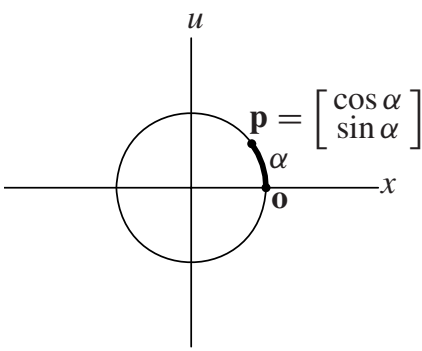

(a)

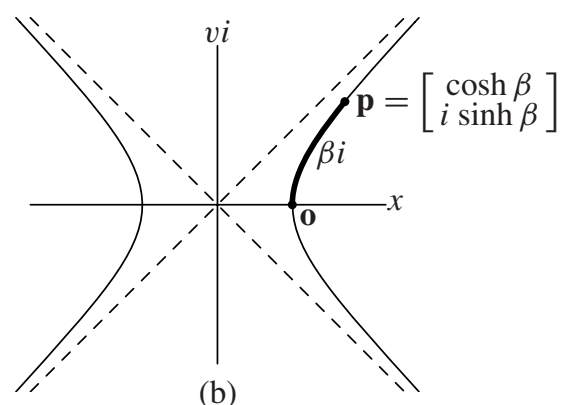

(b)

Figure 5. Real and imaginary radian measure.

Next, start at $\mathbf{o}$, but this time move along the hyperbola on the $x$-vi plane, upward and to the right, to a point $\mathbf{p}$. Because it is on the hyperbola, $\mathbf{p}=\left[\begin{array}{c}\cosh \beta \\ i \sinh \beta\end{array}\right]$ for some positive real number $\beta$, as illustrated in Figure 5(b). The traversed path is the graph of the equation $v=f(x)=\sqrt{x^{2}-1}$, for $1 \leq x \leq \cosh \beta$ on the $x$-vi plane.

We claim the path has length $\beta i$. To show this, partition the interval from 1 to $\cosh \beta$ on the $x$ axis, and set up the Riemann sum for arclength in the usual way, but with (4) replacing the Pythagorean theorem. The length of the path is approximately

$$
\begin{aligned}
L & \approx \sum_{k=1}^{n} \sqrt{\Delta x^{2}+\left[i f\left(x_{k+1}\right)-i f\left(x_{k}\right)\right]^{2}}=\sum_{k=1}^{n} \sqrt{1-\left[f^{\prime}\left(x_{k}^{*}\right)\right]^{2}} \Delta x \\
& =i \sum_{k=1}^{n} \sqrt{\left[f^{\prime}\left(x_{k}^{*}\right)\right]^{2}-1} \Delta x .
\end{aligned}
$$


Since $f^{\prime}(x)>1$, the function $\sqrt{\left[f^{\prime}(x)\right]^{2}-1}$ is real-valued (and continuous away from 1), so taking the limit $\Delta x \rightarrow 0$ completes the claim by giving the length as

$$
L=i \int_{1}^{\cosh \beta} \sqrt{\left[\frac{d}{d x} \sqrt{x^{2}-1}\right]^{2}-1} d x=i \int_{1}^{\cosh \beta} \frac{d x}{\sqrt{x^{2}-1}}=\beta i
$$

Generalizing the idea of real radian measure, we define imaginary radian measure to be the oriented arclength from $\mathbf{o}$ to a point $\mathbf{p}$ on the hyperbola, as illustrated in Figure 5(b). If $\mathbf{p}$ is above the $x$ axis, its radian measure is $\beta i$ with $\beta>0$, while if it is below the $x$ axis, its radian measure is $\beta i$ with $\beta<0$. As in the real case, we define $\cos \beta i$ and $\sin \beta i$ to be the $z$ and $w$ coordinates of p. According to Figure 5(b), this gives

$$
\begin{aligned}
& \cos \beta i=\cosh \beta \\
& \sin \beta i=i \sinh \beta
\end{aligned}
$$

Notice that (5) and (6) hold for both positive and negative values of $\beta$, and are in agreement with (1) and (2).

\section{Complex radian measure}

One can begin to understand complex radian measure from Figure 1. Begin at the point o, and move counterclockwise a distance of $\pi$ along the circle on the real $x$ - $u$ plane to the point $\mathbf{- o}$. Then move down along the hyperbola an imaginary distance of $\beta i$ to the point $\mathbf{p}=\left[\begin{array}{c}-\cosh \beta \\ -i \sinh \beta\end{array}\right]$. This covers a total complex distance of $\pi+\beta i$. Interpreting cos and $\sin$ as the $z$ and $w$ coordinates of $\mathbf{p}$ gives

$$
\begin{aligned}
& \cos (\pi+\beta i)=-\cosh \beta \\
& \sin (\pi+\beta i)=-i \sinh \beta,
\end{aligned}
$$

in agreement with (1) and (2). Visualizing complex radians where the real part is not a multiple of $\pi$ takes a little more care, though it is the same in spirit.

It is helpful to observe that (3) can be factored as

$$
\mu(\alpha+\beta i)=\left[\begin{array}{cc}
\cos \alpha & -\sin \alpha \\
\sin \alpha & \cos \alpha
\end{array}\right]\left[\begin{array}{c}
\cosh \beta \\
i \sinh \beta
\end{array}\right]
$$

Set $M_{\alpha}=\left[\begin{array}{cc}\cos \alpha & -\sin \alpha \\ \sin \alpha & \cos \alpha\end{array}\right]$. It is easy to check that the linear transformation $M_{\alpha}: \mathbb{C}^{2} \rightarrow$ $\mathbb{C}^{2}$ preserves complex distance, that is, $d\left(M_{\alpha} \mathbf{x}, M_{\alpha} \mathbf{y}\right)=d(\mathbf{x}, \mathbf{y})$ for all $\mathbf{x}$ and $\mathbf{y}$ in $\mathbb{C}^{2}$.

Now we can understand what is meant by complex radian measure $\alpha+\beta i$. For the moment, let $\alpha$ and $\beta$ be positive and consider the point $\alpha+\beta i$ in the complex plane. We can get from 0 to $\alpha+\beta i$ by first moving along the path $P_{1}, \gamma(t)=t$, for $0 \leq t \leq \alpha$, and then along path $P_{2}, \delta(t)=\alpha+t i$, for $0 \leq t \leq \beta$, as illustrated in Figure 6 . This figure also illustrates how $\mu$ sends $P_{1}$ and $P_{2}$ to curved paths on $U$ that combine to form a path on $U$ from $\mathbf{o}$ to $\mathbf{p}=\mu(\alpha+\beta i)$. The image of $P_{1}$ has length $\alpha$ since it lies along the unit circle in the $x$ - $u$ plane. By (7), the image of $P_{2}$ can be written as $M_{\alpha}\left[\begin{array}{c}\cosh t \\ i \sinh t\end{array}\right]$, for $0 \leq t \leq \beta$, and its length is $\beta i$ because it has the form of an isometry applied to a path of length $\beta i$. Therefore, in going from $\mathbf{o}$ to $\mathbf{p}$, we have gone 

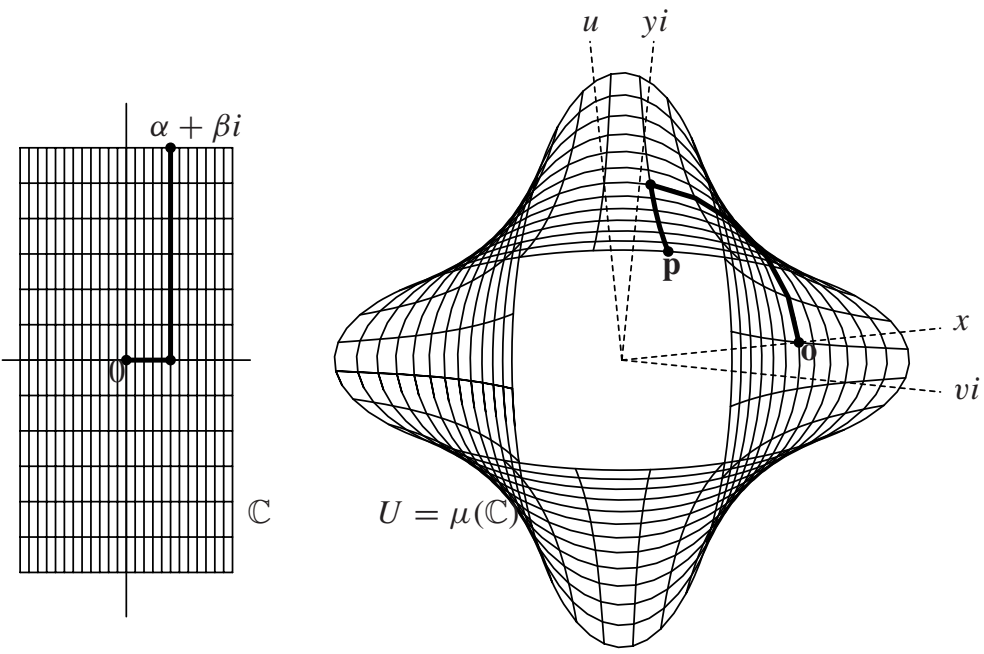

Figure 6. Complex radian measure $\alpha+\beta i$.

a total distance of $\alpha+\beta i{ }^{1}$ Defining $\cos (\alpha+\beta i)$ and $\sin (\alpha+\beta i)$ to be the $z$ and $w$ coordinates of $\mathbf{p}=\mu(\alpha+\beta i)$, we get (1) and (2) from (3).

If $\alpha$ and $\beta$ are not both positive, then careful attention to the branch of the square root function we are using shows that the distance from $\mathbf{o}$ to $\mathbf{p}=\mu(\alpha+\beta i)$ along $U$ is $|\alpha|+|\beta| i$. This is like the situation of the traditional (real) unit circle, where the arclength along the circle between radians 0 and $\alpha$ is $|\alpha|$.

Here, then, is a summary of how one can visualize trigonometric functions of a complex variable. Use $\mu$ to coordinatize the complex unit circle $U$. Any number $\alpha+$ $\beta i$ in $\mathbb{C}$ is identified with the point $\mathbf{p}=\mu(\alpha+\beta i)$ on $U$, which is at complex distance $|\alpha|+|\beta| i$ along $U$ from $\mathbf{o}$. Function values $\cos (\alpha+\beta i)$ and $\sin (\alpha+\beta i)$ are the $z$ and $w$ coordinates of $\mathbf{p}$.

\section{Complex angles}

To conclude our excursion, we explore the idea of angles in $\mathbb{C}^{2}$. This entails examining complex versions of lines and rays. For simplicity, we consider only lines and rays through the origin $\mathbf{0}$, and consequently only angles whose vertex is at $\mathbf{0}$.

Since a line (through the origin) in $\mathbb{R}^{2}$ is the span of a nonzero vector, we define a complex line in $\mathbb{C}^{2}$ analogously. For a nonzero vector $\mathbf{u}$ in $\mathbb{C}^{2}$, the complex line $\Lambda$ through $\mathbf{u}$ is $\operatorname{span}(\mathbf{u})$, which is isomorphic to the complex plane.

There are two special complex lines in $\mathbb{C}^{2}$ :

$$
\begin{aligned}
& O^{+}=\operatorname{span}\left(\left[\begin{array}{l}
1 \\
i
\end{array}\right]\right)=\left\{\left[\begin{array}{c}
\lambda \\
i \lambda
\end{array}\right] \mid \lambda \in \mathbb{C}\right\} \text { and } \\
& O^{-}=\operatorname{span}\left(\left[\begin{array}{c}
1 \\
-i
\end{array}\right]\right)=\left\{\left[\begin{array}{c}
\lambda \\
-i \lambda
\end{array}\right] \mid \lambda \in \mathbb{C}\right\} .
\end{aligned}
$$

\footnotetext{
${ }^{1}$ There are, of course, other paths from $\mathbf{o}$ to $\mathbf{p}$ on $U$, and they all have length $\alpha+\beta i$, under complex distance: Consider path $x(t)+y(t) i, 0 \leq t \leq 1$ from 0 to $\alpha+\beta i$ on $\mathbb{C}$. It is instructive to show that by employing (4) in the standard limit process for arc length, the image of this path on $U$ has length $\int_{0}^{1} \sqrt{\left(\frac{d}{d t}[\cos (x(t)+y(t) i)]\right)^{2}+\left(\frac{d}{d t}[\sin (x(t)+y(t) i)]\right)^{2}} d t=\int_{0}^{1} \sqrt{\left(x^{\prime}(t)+y^{\prime}(t) i\right)^{2}} d t$. If $x^{\prime}(t)+y^{\prime}(t) i$ never crosses the negative real axis (where the square root is discontinuous) this integral is $\int_{0}^{1}\left(x^{\prime}(t)+y^{\prime}(t) i\right) d t=$ $\alpha+\beta i$, as claimed. If $x^{\prime}(t)+y^{\prime}(t) i$ does cross the negative real axis, the integral may be done piecewise, alternatively adding and subtracting, and the answer will still be $\alpha+\beta i$.
} 
Any two points on either of these complex lines are at complex distance of 0 apart, and hence neither of these lines meets $U$. (The asymptotes in Figure 4 are where $O^{+}$and $O^{-}$intersect the $x$-vi plane, so $U$ is evidently asymptotic to these two complex lines.) In fact, these are the only two complex lines (through the origin) that do not meet $U$. As is easily checked, any line $\Lambda=\operatorname{span}\left(\left[\begin{array}{c}z_{0} \\ w_{0}\end{array}\right]\right)$ that is neither $O^{+}$nor $O^{-}$meets $U$ exactly at the two points

$$
\frac{ \pm 1}{\sqrt{z_{0}^{2}+w_{0}^{2}}}\left[\begin{array}{c}
z_{0} \\
w_{0}
\end{array}\right] .
$$

In $\mathbb{R}^{2}$, the ray $\overrightarrow{\mathbf{u}}$ passing through a nonzero vector $\mathbf{u}$ can be defined as the set of all nonnegative real multiples of $\mathbf{u}$. Extending this to $\mathbb{C}^{2}$ seems problematic, for the word "nonnegative" has no meaning in $\mathbb{C}$. Using the half-plane $H$ (Figure 3 ) seems a reasonable alternative. If $\mathbf{u}$ is a nonzero vector in $\mathbb{C}$, then the complex ray through $\mathbf{u}$ is the set $\overrightarrow{\mathbf{u}}=\{\lambda \mathbf{u} \mid \lambda \in H\}$. If $\mathbf{u}$ is in neither $O^{+}$nor $O^{-}$, the considerations of the previous paragraph imply that $\overrightarrow{\mathbf{u}}$ meets $U$ in exactly one point.

A complex angle is the union of two complex rays $\overrightarrow{\mathbf{u}}_{1}$ and $\overrightarrow{\mathbf{u}}_{2}$. If the rays are distinct, they intersect only at $\mathbf{0}$. If neither is in $O^{+} \cup O^{-}$, then they meet $U$ at points $\mu\left(\theta_{1}\right)$ and $\mu\left(\theta_{2}\right)$, and, as in the real case, we say the angle has radian measure equal to the complex number $\theta_{2}-\theta_{1}$, which is the oriented distance along $U$ from $\mu\left(\theta_{1}\right)$ to $\mu\left(\theta_{2}\right)$.

In this way, we can visualize any complex angle, and, provided neither of its rays is in $\mathrm{O}^{+} \cup \mathrm{O}^{-}$, understand radian measure of angles in $\mathbb{C}^{2}$ in a manner entirely analogous to the familiar setting of $\mathbb{R}^{2}$. In conclusion, we show that at least one familiar formula from $\mathbb{R}^{2}$ carries over to our wider view of complex trigonometric functions.

Define the complex dot product of vectors $\mathbf{u}=\left[\begin{array}{l}z_{1} \\ w_{1}\end{array}\right]$ and $\mathbf{v}=\left[\begin{array}{c}z_{2} \\ w_{2}\end{array}\right]$ in $\mathbb{C}^{2}$ to be $\mathbf{u} \cdot \mathbf{v}=z_{1} z_{2}+w_{1} w_{2}$, and the complex length of $\mathbf{u}$ to be $|\mathbf{u}|=\sqrt{\mathbf{u} \cdot \mathbf{u}}$. We take care to point out that the complex dot product differs from the standard unitary dot product $\mathbf{u} \cdot \mathbf{v}=z_{1} \overline{z_{2}}+w_{1} \overline{w_{2}}$ (as defined, for example, in [2, p. 271]). In fact, it violates several of the formal inner product axioms, so it is not an inner product. Also, although $|\mathbf{u}|$ equals the complex distance from $\mathbf{0}$ to $\mathbf{u}$, it is not a norm in the technical sense of the word. Still, defining things this way is meaningful in the context of trigonometric functions as we have developed them.

Suppose $\mathbf{u}$ and $\mathbf{v}$ are not in $O^{+} \cup O^{-}$, so $|\mathbf{u}|$ and $|\mathbf{v}|$ are nonzero. The complex rays $\overrightarrow{\mathbf{u}}$ and $\overrightarrow{\mathbf{v}}$ meet $U$ at points $\frac{\mathbf{u}}{|\mathbf{u}|}$ and $\frac{\mathbf{v}}{|\mathbf{v}|}$, respectively, so there are complex numbers $\theta_{1}$ and $\theta_{2}$ with $\frac{\mathbf{u}}{|\mathbf{u}|}=\left[\begin{array}{c}\cos \theta_{1} \\ \sin \theta_{1}\end{array}\right]$ and $\frac{\mathbf{v}}{|\mathbf{v}|}=\left[\begin{array}{c}\cos \theta_{2} \\ \sin \theta_{2}\end{array}\right]$. Thus the radian measure of the angle formed by $\mathbf{u}$ and $\mathbf{v}$ is $\theta=\theta_{2}-\theta_{1}$. Since $\frac{\mathbf{u}}{|\mathbf{u}|} \cdot \frac{\mathbf{v}}{|\mathbf{v}|}=\cos \theta_{1} \cos \theta_{2}+\sin \theta_{1} \sin \theta_{2}=\cos \left(\theta_{2}-\theta_{1}\right)=$ $\cos \theta$, we obtain

$$
\mathbf{u} \cdot \mathbf{v}=|\mathbf{u}||\mathbf{v}| \cos \theta .
$$

This expression—familiar as an identity in $\mathbb{R}^{2}$ (or $\mathbb{R}^{n}$ )— has no meaning in the unitary space $\mathbb{C}^{2}$, but it fits neatly into our model of complex trigonometric functions.

Acknowledgments. I thank the Editor for carefully reading several drafts and suggesting innumerable improvements. Thanks also to Dorothee Blum and John Weiss who directed my master's thesis [1] that contained an early version of these results. And thanks to the late David Brisson, whose conversations inspired this work.

\section{References}

1. R. Hammack, Geometry of Complex Vector Spaces, Master's Thesis, Virginia Commonwealth University, 1988.

2. K. Hoffman and R. Kunze, Linear Algebra (2nd ed.), Prentice-Hall, 1971.

3. B. Palka, An Introduction to Complex Function Theory (1st ed.), Springer-Verlag, 1991. 